\title{
Simultaneous multiple wavelength operation of a multistripe array grating integrated cavity laser
}

\author{
K. R. Poguntke, ${ }^{\text {a) }}$ J. B. D. Soole, A. Scherer, H. P. LeBlanc, C. Caneau, R. Bhat, \\ and M. A. Koza \\ Bellcore, 331 Newman Springs Road, Red Bank, New Jersey 07701
}

(Received 20 October 1992; accepted for publication 1 February 1993)

\begin{abstract}
Simultaneous multiple-wavelength operation of a multistripe array grating integrated cavity laser is reported. We demonstrate simultaneous lasing from a single output port at 2,3 , and 4 discrete wavelengths, each independently selected from a comb of 9 wavelengths set at $\sim 2 \mathrm{~nm}$ intervals, from 1512 to $1528 \mathrm{~nm}$. Signal cross talk is examined for the case of two-wavelength emission and found to be independent of the wavelength separation. The emission wavelengths were linearly spaced to within $\pm 0.02 \mathrm{~nm}$, while the comb's central wavelength deviated by less than $2 \mathrm{~nm}$ from its design value. This is the highest wavelength linearity and accuracy so far recorded for a monolithic multiwavelength source.
\end{abstract}

Wavelength division multiplexing (WDM) is being widely considered for use in future fiber networks by both the telecommunications and computer industries. ${ }^{1}$ Many of the proposed networks call for multiple-wavelength sources at the access points (and nodes) within the network. System integrity requires that all such sources provide signals at the same precisely defined wavelengths.

Banks of independent distributed feedback (DFB) lasers have already been used in WDM network demonstrations, their different wavelength outputs being combined in an external bulk-optic multiplexer to form a single multiwavelength source. ${ }^{2-4}$ Integration of these separate lasers on a single chip potentially offers great advantage through reduced component count and packaging costs, as well as through improved reliability. ${ }^{5,6}$ However, integration removes the ability to independently temperature tune the wavelengths of the individual laser elements. Very precise control of the growth and fabrication of each element is therefore necessary in order to accurately set all wavelengths.

We have recently reported a new type of semiconductor laser source, the multistripe array grating integrated cavity (MAGIC) laser. ${ }^{7,8}$ We have already demonstrated single-wavelength operation of this device, showing that lasing can occur at a wavelength selected from a predetermined comb of values. ${ }^{7,8}$ In this letter we report the first demonstration of simultaneous multiwavelength operation of a MAGIC laser. Simultaneous operation at 2, 3, and 4 wavelengths is reported, each wavelength being independently selected from a comb of 9 wavelengths spaced at $\sim 2$ $\mathrm{nm}$ intervals across the spectral range of 1512-1528 nm. The multiple-wavelength emission occurs from a single output port. Record precision of wavelength positioning is also demonstrated. Finally, we examine signal cross talk for the case of two-wavelength operation and show that it is approximately independent of the wavelength spacing.

A MAGIC laser is formed by the monolithic integration of an active stripe array with a diffraction grating, the latter being etched into one end of a two-dimensional pla-

${ }^{a j}$ Visitor from Department of Physics, University of Surrey, UK. nar waveguide. The operation of the MAGIC laser resembles that of a bulk-optic external cavity laser, for which the single laser element and rotating grating of the latter have been replaced by an array of laser stripes and a fixed etched grating, the etched grating being curved to provide focusing as well as diffraction. The MAGIC laser is shown schematically in Fig. 1.

If a single active stripe of the MAGIC laser is electrically pumped, laser emission occurs from its cleaved end facet at a wavelength determined by the optical feedback from the grating. The lasing wavelength is different for each stripe, being defined by the stripe's position relative to the grating. ${ }^{7}$ Laser emission at different wavelengths from a single output port is also possible. An "output" stripe toward one end of the array is selected and pumped, and a "second" stripe toward the opposite end of the array is also pumped. If the two stripes are chosen such that the direct grating-feedback wavelengths for each occur at opposite extremes of the active material gain spectrum, then the wavelength at which the grating "connects" the two lies toward the middle of this spectrum, and so lasing occurs preferentially at the connecting wavelength. ${ }^{8}$ By then, pumping different second stripes, the emission from the output stripe may be switched from one wavelength to another. In this letter, we report the simultaneous operation of the MAGIC laser at a number of different wavelengths, achieved by simultaneously injection pumping a number of different second stripes together with the single output stripe. Each emission wavelength is determined by the resonance between the output stripe and one second

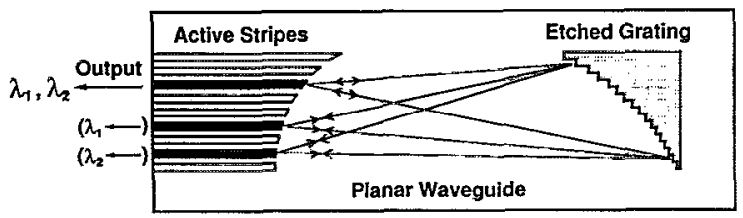

FIG. 1. Sketch of the MAGIC laser. Shaded stripes illustrate those injection pumped for simultaneous multiwavelength operation; laser emission is collected from the common output stripe. 


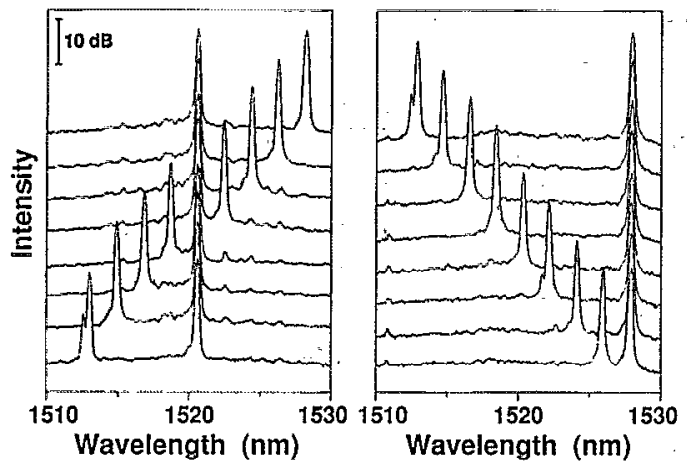

FIG. 2. Simultaneous emission at two wavelengths selected from the comb of wavelengths available from the MAGIC laser. Different twowavelength combinations are shown, illustrating emission across a $16 \mathrm{~nm}$ wavelength range. Scans were taken with a $0.1 \mathrm{~nm}$ rcsolution optical spectrum analyzer, the output stripe current was $200 \mathrm{~mA}$, and the second stripe currents were $150 \mathrm{~mA}$; active stripes were $1500 \mu \mathrm{m}$ by $7 \mu \mathrm{m}$.

stripe. The pumping arrangement is shown schematically in Fig. 1.

The structure and fabrication of the MAGIC laser used is identical to that described in Ref. 8, and will not be discussed in detail here. In brief, the structure consists of an $\operatorname{InP}(1.0 \mu \mathrm{m}) / \operatorname{InGaAsP}\left(0.3 \mu \mathrm{m}, \lambda_{\mathrm{g}}=1.3 \mu \mathrm{m}\right) / \mathrm{InP}$ planar waveguide grown on a $n^{+}$-InP substrate, with a curved diffraction grating etched vertically through the guide core at one end, and an array of $\sim 40-\mu \mathrm{m}$-spaced active stripes at the other. The grating is used in 16th order, giving a free spectral range of $\sim 90 \mathrm{~nm}$ for operation around $1.5 \mu \mathrm{m}$. The active region of the stripes comprises a 6-well unstrained InGaAs/InGaAsP $\left(\lambda_{g}=1.3 \mu \mathrm{m}\right)$ multi-quantumwell stack grown directly on top of the waveguide core. Patterning of the active stripes is followed by regrowth of a $1.0 \mu \mathrm{m}$. InP waveguide cladding layer, made semiinsulating to provide diode isolation as well as surface passivation. The laser was mounted on a temperaturecontrolled stage, and was operated in pulsed mode.

The spectrum obtained for simultaneous twowavelength operation is illustrated in Fig. 2. The bias for the output stripe was held constant at $200 \mathrm{~mA}$ while different combinations of second stripes were pumped; injection currents of $\sim 90 \mathrm{~mA}$ were required to reach lasing threshold. The traces in the figure were obtained with second stripe injection currents of $\sim 150 \mathrm{~mA}$, i.e., $\sim 1.6 I_{\mathrm{th}}$. The active stripes were $\sim 1500-\mu \mathrm{m}$ long by $\sim 7-\mu \mathrm{m}$ wide. Two series of scans are shown, illustrating a number of possible combinations from a comb of 9 wavelengths spaced at $\sim 2 \mathrm{~nm}$ from 1512 to $1528 \mathrm{~nm}$.

All laser intensities lie $\sim 20 \mathrm{~dB}$ above the background of spontaneous emission. There was no evidence of laser emission at the output stripe's own direct grating-feedback wavelength. This is the result of using a stripe for which the direct grating-feedback wavelength lies well into the wings of the gain spectrum of the active material. Indeed, for the case illustrated, direct grating feedback to the output stripe occurred at a wavelength close to the band gap of the active material. We also note the absence of features associated with Fabry-Perot resonances which might arise
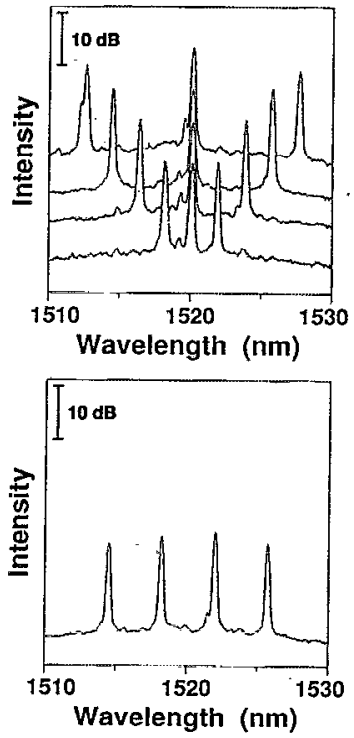

FIG. 3. Simultaneous laser operation at 3 and 4 emission wavelengths. Same pumping conditions as for the traces in Fig. 4 .

from residual reflections at the interface between the active stripes and the planar waveguide. We do notice in Figs. 2 and 3 the presence of residual peaks in the background emission spectrum at wavelengths that correspond to some of the laser emissions; their origin is presently unclear.

The linearity of the wavelength spacing depicted in Fig. 2 is high. The standard deviation is just $0.02 \mathrm{~nm}$. This is less than the longitudinal mode spacing $(\sim 0.03 \mathrm{~nm})$, and suggests that wavelength uniformity is achieved to the nearest longitudinal mode. It is interesting to note that a wavelength tolerance of $\sim 0.02 \mathrm{~nm}$ is expected from considerations of fabrication and mask dimensional tolerances. This degree of wavelength uniformity is unrivaled for a monolithic laser source and arises from the nature of the wavelength selection mechanism; comparable wavelength precision from a conventional DFB laser would require grating accuracies on the order of $0.003 \mathrm{~nm}$. Absolute emission wavelengths differed by just $\sim 2 \mathrm{~nm}$ from their design values. Exact coincidence was obtained by adjusting the temperature of the laser mount. The measured temperature sensitivity was $0.11 \mathrm{~nm} /{ }^{\circ} \mathrm{C}$.

Figure 3 shows simultaneous laser operation at three and four wavelengths. The same output stripe was used, but now three or four second stripes were pumped. Again, the bias current for the output stripe was $200 \mathrm{~mA}$, and second stripe injection currents were $\sim 150 \mathrm{~mA}$. As for the two-wavelength case, laser intensities are found to lie between 15 and $20 \mathrm{~dB}$ above a featureless background of spontaneous emission. Simultaneous operation on an even greater number of wavelengths is expected to give similar results.

We have examined interchannel cross talk for the case of two-wavelength opcration. We measured the change of output power $P_{1}$ at a single wavelength that occurs as a result of increased power $P_{2}$ at a second, different, wavelength. The intensity of the first wavelength emission in the absence of any second wavelength emission is denoted $P_{10}$. 


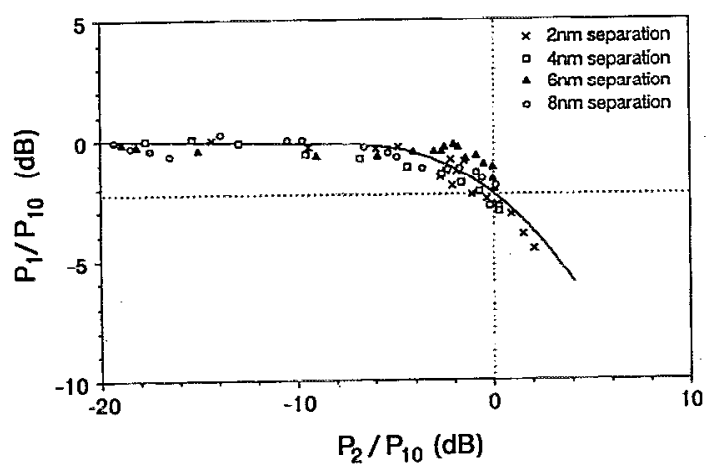

FIG. 4. Cross talk for the case of two-wavelength operation. Relative output power at one wavelength, $P_{1} / P_{10}$, as a function of output power at another, $P_{2} / P_{10}$, separated from the first by $2,4,6$, and $8 \mathrm{~nm}$.

In Fig. 4 we plot the relative power of the first wavelength cmission $P_{1} / P_{10}$ against the relative power $P_{2} / P_{10}$ of the second. The injection currents for the output and first second stripe were maintained at 200 and $150 \mathrm{~mA}$, respectively. Data points are shown for four different wavelength separations: $2,4,6$, and $8 \mathrm{~nm}$.

The steady-state output power at one wavelength is seen to decrease with increasing power at the second wavelength; a $\sim 2 \mathrm{~dB}$ reduction occurs for a power of $P_{10}$ at the second wavelength. The effect is approximately independent of the channel spacing. This behavior is consistent with interaction via gain saturation in the common active output stripe. The gain saturation mechanism has previously been studied for multichannel amplification by traveling-wave amplifiers, ${ }^{9-11}$ where the level of gain supprcssion was found to depend upon the total intensity within the active material rather than upon the intensity at any single wavelength. ${ }^{9}$ Typical operation of the MAGIC laser (such as that shown in Fig. 2) gives instantaneous output powers of order $1 \mathrm{~mW}$, and such high optical intensities within the output stripe suppress the material gain relative to the small-signal value. ${ }^{9,10}$ Figure 4 indicates a homogeneous gain saturation mechanism where increasing the power at any one wavelength affects the material gain at all wavelengths.

Cross talk of comparable magnitude to that found here has been recently predicted ${ }^{12}$ and also experimentally demonstrated $^{13}$ for a bulk optic laser similar to the integrated MAGIC laser. The former device comprised a bar of active stripes with one facet antireflection coated, an external collimating lens, and a plane grating. For this laser, it was shown that the cross talk could be compensated by super- imposing a component of the injection current to each second stripe onto the injection current to the common output stripe. ${ }^{13}$ Cross-talk-induced power reductions of less than $0.05 \mathrm{~dB}$ were demonstrated. ${ }^{13}$ Similar performance may be expected for the MAGIC laser.

In summary, we have reported the first demonstration of simultancous multiwavelength operation of a MAGIC laser. Simultaneous emission at 2, 3, and 4 wavelengths was demonstrated from a single output, with each wavelength independently selected from an array of highly linearly spaced $(\sigma=0.02 \mathrm{~nm})$ wavelengths, set at $\sim 2 \mathrm{~nm}$ intervals. A change in operating temperature of $\pm 9{ }^{\circ} \mathrm{C}$ allows the comb of emission wavelengths to be placed anywhere within the spacing interval of $2 \mathrm{~nm}$. This represents the highest wavelength precision so far reported for a monolithic WDM source. Interchannel cross talk was also measured for two-wavelength operation and found to be independent of channel spacing. This cross talk may be reduced to a negligible level by appropriate current injection schemes.

We thank B. Pathak and E. Goldstein for helpful discussions, P. J. Rickman for graphical assistance, and B. Pathak, D. A. Smith, S. J. B. Yoo, A. Weiner, and G.-K. Chang for the loan of equipment. One of us (K.R.P.) wishes to thank United Kingdom S.E.R.C. for financial support.

${ }^{1}$ C. Brackett, J. Sel. Arcas Comm, 8, 948 (1990).

${ }^{2}$ N. A. Olsson, J. Hegarty, R. A. Logan, L. F. Johnson, K. L. Walker, L.

G. Cohen, B. L. Kasper, and J. C. Campbell, Electron. Lett. 21, 105 (1985).

${ }^{3}$ S. S. Wagner and H. L. Lemberg, IEEE J. Lightwave Technol. LT-7, 1759 (1989).

${ }^{4}$ P. J. Chidgey and G. R. Hill, Electron. Lett. 26, 1709 (1990).

${ }^{5}$ C. E. Zah, P. S. D. Lin, F. Favire, B. Pathak, R. Bhat, C. Caneau, A. Gozdz, N. C. Andreadakis, M. A. Koza, and T. P. Lee, Electron. Lett. 29, 824 (1992).

${ }^{6}$ C. E. Zah, F. J. Favire, B. Pathak, C. Caneau, P. S. D. Lin, A. S. Gozdz, N. C. Andreadakis, M. A. Koza, and T. P. Lee, Proceedings of the 13th International Semiconductor Conference, Kagawa, Japan, 21-25 September, 1992 (unpublished), paper K-1.

${ }^{7}$ J. B. D. Soole, K. Poguntke, A. Scherer, H. P. LeBlanc, C. ChangHasnain, J. R. Hayes, R. Bhat, C. Caneau, and M. A. Koza, Electron. Lett. 28, 1805 (1992).

${ }^{8}$ J. B. D. Soole, K. Poguntke, A. Scherer, H. P. LeBlanc, C. ChangHasnain, J. R. Hayes, R. Bhat, C. Caneau, and M. A. Koza, Appl. Phys. Lett. 61, 2750 (1992).

${ }^{9}$ T. Mukai, K. Inoue, and T. Saitoh, Electron. Lett. 23, 396 (1987).

${ }^{10}$ T. Saitoh and T. Mukai, IEEE J. Lightwave Technol. LT-6, 1656 (1988).

${ }^{11} \mathrm{~K}$. Inoue, IEEE J. Lightwave Technol. LT-7, 1118 (1989).

${ }^{12}$ I. H. White, IEEE J. Lightwave Technol. LT-9, 893 (1991).

${ }^{13}$ K. O. Nyairo, I. H. White, C. J. Armistead, and P. A. Kirkby, Electron. Lett. 28, 261 (1992). 